ISSN 1855-3966 (printed edn.), ISSN 1855-3974 (electronic edn.)

ARS MATHEMATICA CONTEMPORANEA 20 (2021) 243-260

https://doi.org/10.26493/1855-3974.2359.a7b

(Also available at http://amc-journal.eu)

\title{
Wiener-type indices of Parikh word representable graphs*
}

\author{
Nobin Thomas \\ Research scholar, APJ Abdul Kalam Technological University, \\ Thiruvananthapuram, Kerala 695016 India, and \\ Amal Jyothi College of Engineering, Kanjirappally, Kerala 686518 India \\ Lisa Mathew \\ Amal Jyothi College of Engineering, Kanjirappally, Kerala 686518 India

\section{Sastha Sriram} \\ Department of Mathematics, School of Arts, Science and Humanities, \\ SASTRA Deemed University, Tanjore, Tamil Nadu 613401 India \\ K. G. Subramanian ${ }^{\dagger}$ \\ School of Mathematics, Computer Science and Engineering, \\ Liverpool Hope University, Liverpool L16 9JD, United Kingdom
}

Received 10 June 2020, accepted 14 February 2021, published online 9 November 2021

\begin{abstract}
A new class of graphs $G(w)$, called Parikh word representable graphs ( $P W R G)$, corresponding to words $w$ that are finite sequence of symbols, was considered in the recent past. Several properties of these graphs have been established. In this paper, we consider these graphs corresponding to binary core words of the form $a u b$ over a binary alphabet $\{a, b\}$. We derive formulas for computing the Wiener index of the $P W R G$ of a binary core word. Sharp bounds are established on the value of this index in terms of different parameters related to binary words over $\{a, b\}$ and the corresponding PWRGs. Certain other Wienertype indices that are variants of Wiener index are also considered. Formulas for computing these indices in the case of $P W R G$ of a binary core word are obtained.
\end{abstract}

Keywords: Graphs, words, Parikh matrix, Parikh word representable graphs.

Math. Subj. Class. (2020): 68R10, 68R15

\footnotetext{
* The authors would like to thank the reviewers for their very useful comments which enabled them to revise the paper improving the presentation of the paper.

${ }^{\dagger}$ Honorary Visiting Professor.

E-mail addresses: nobinvazhayil@gmail.com (Nobin Thomas), lisamathew@amaljyothi.ac.in (Lisa Mathew), sriram.discrete@gmail.com (Sastha Sriram), kgsmani1948@gmail.com (K. G. Subramanian)
} 


\section{Introduction}

While words that are finite sequences of symbols are the fundamental and central objects in computing models developed in theoretical studies of computer science, graphs are mathematical models of pairwise relations between objects found useful for analyzing and solving different kinds of problems. An interesting area of investigation is relating graphs and words and there are many studies in this direction (see, for example, [7, 10, 15, 18, 19, 24]).

On the other hand, in the study of numerical quantities related to subwords (also called scattered subwords) of a word, the notion of Parikh matrix of a word over an ordered alphabet was introduced in [26]. This has opened up a new direction of research in the area of combinatorics on words [23] and many problems on words and subwords have been investigated (see, for example, [1, 2, 4, 25, 33, 34, 36, 37, 38] and references therein), resulting in a number of interesting results. Parikh word representable graph $(P W R G)$ is one such notion introduced in [3] linking the two areas of study on properties of words and of graphs. Based on the notions of subwords of a word and the Parikh matrix of a word [26] with entries of the matrix giving the counts of certain subwords in the word, $P W R G$ related to a word was introduced in [3]. Relationship of these graphs with corresponding words and partitions was recently studied in [27].

In the field of chemical graph theory [14], undirected graphs, referred to as molecular graphs are considered providing graph representations of organic compounds or equivalently their molecular structures with atoms other than hydrogen often represented by vertices and covalent chemical bonds by edges. In fact in chemical graph theory there have been attempts to capture the molecular structure in terms of the topological index of the corresponding graph. There has been a great interest in various topological indices associated with graphs due to their application in the area of chemical graph theory [8]. There are a number of studies (see, for example, [14]) of various topological indices of graphs establishing formulae for computing the indices and also providing upper and lower bounds on the values of such indices. The Wiener index (also called Wiener number) [40] is the first topological index introduced by Harold Wiener. Knor et al. [22] provide an excellent summary of results relating to Wiener index besides providing conjectures and problems on this index. Wiener index and its variants for different classes of graphs are widely investigated indices (see, for example, [9, 12, 14, 21, 28, 29, 39] and references therein).

In this paper we study the Wiener index of a $P W R G$ of a binary core word and derive formulas for computing this index besides establishing sharp bounds on their values, given different parameters related to the graphs. We also obtain formulas for evaluating certain other indices that are variants of Wiener index, such as multiplicative Wiener index [13], terminal Wiener index [11], peripheral Wiener index [16], hyper-Wiener index [20, 30] in the case of a $P W R G$ of a binary core word.

\section{Preliminaries}

The basic notions and notations relating to words and subwords can be found in [23, 31]. We recall some essential concepts and results. An ordered alphabet $\Sigma$ which is a set of symbols $\left\{a_{1}, a_{2}, \ldots, a_{s}\right\}$ with an ordering $<$ on its symbols is written as $\Sigma=$ $\left\{a_{1}<a_{2}<\cdots<a_{s}\right\}$. A word $v$ is a subword of a word $w$ over $\Sigma$ if and only if we can find words $x_{1}, x_{2}, \ldots, x_{n}, y_{0}, y_{1}, \ldots, y_{n}$ over $\Sigma$, some of them possibly empty, such that $w=y_{0} x_{1} y_{1} x_{2} y_{2} \cdots y_{n-1} x_{n} y_{n}$ and $v=x_{1} x_{2} \cdots x_{n}$. The number of occurrences of a word $u$ as a subword of $w$ is denoted by $|w|_{u}$. For example, in the word $w=a a b a b a a a b=$ 
$a^{2} b a b a^{3} b$ over the ordered binary alphabet $\Sigma=\{a<b\}$, the number of distinct occurrences of the subword $a b$ is 11 so that $|w|_{a b}=11$. The set of all words over an alphabet $\Sigma$, including the empty word $\lambda$ with no symbols, is denoted by $\Sigma^{*}$.

Definition 2.1 ([6]). A binary word $w$ over an alphabet $\{a, b\}$ is said to be fair if $|w|_{a b}=|w|_{b a}$.

Example 2.2. The binary word $a b b b a a b$ is a fair word since $|w|_{a b}=|w|_{b a}=6$.

Definition 2.3 ([38]). Consider the binary word $w \in \Sigma^{*}$ where $\Sigma=\{a<b\}$. The core of $w$, denoted by core $(w)$, is the unique word $w_{0}$ of $w$ with the smallest possible length such that $w \in b^{*} w_{0} a^{*}$. A word $w \in \Sigma^{*}$ is said to be a core word if and only if $\operatorname{core}(w)=w$.

Clearly, a non empty word $w$ over $\Sigma=\{a<b\}$ is a core word if and only if $w$ starts with $a$ and ends with $b$.

We now recall the relationship between binary core words and partitions following the discussion in [38, pages 62-63].

Lemma 2.4 ([38]). Every nonempty binary core word can be identified with a partition of a positive integer.

Proof. Suppose $w \in \Sigma^{*}$ is a nonempty core word and has the form $a^{n_{1}} b a^{n_{2}} b \cdots a^{n_{|w|_{b}}} b$ where $n_{1} \geq 1$ and $n_{k}$ is nonnegative for each $k, 2 \leq k \leq|w|_{b}$. Thus $w$ can be identified with the partition

$$
|w|_{a b}=\left(n_{1}+n_{2}+\cdots+n_{|w|_{b}}\right)+\cdots+\left(n_{2}+n_{1}\right)+n_{1} .
$$

Clearly, distinct core words are identified with distinct partitions.

Conversely, suppose that $m_{1}+m_{2}+\cdots+m_{l}$ is a partition of some positive integer, where $m_{1} \geq m_{2} \geq \cdots \geq m_{l} \geq 1$. It is clear that the word

$$
w=a^{m_{l}} b a^{m_{l-1}-m_{l}} b a^{m_{l-2}-m_{l-1}} b \cdots a^{m_{1}-m_{2}} b
$$

can be identified with the given partition.

We shall use the notation $p(w)=\left(n_{1}+n_{2}+\cdots+n_{l}\right)+\left(n_{1}+n_{2}+\cdots+n_{l-1}\right)+\cdots+$ $\left(n_{1}+n_{2}+\cdots+n_{l-i+1}\right)+\cdots+\left(n_{1}+n_{2}\right)+\left(n_{1}\right)$ to indicate the partition corresponding to the word $w=a^{n_{1}} b a^{n_{2}} b \cdots a^{n_{l}} b$.

We now recall the notion of Parikh word representable graph $(P W R G)$ [3]. For basic concepts pertaining to graphs we refer to [5].

Definition 2.5 ([3]). For a word $w=a_{1} a_{2} \cdots a_{n}$ of length $n$ where for $1 \leq i \leq n$, $a_{i} \in \Sigma=\{a<b\}$, we associate a simple graph $G=G(w)$ with $n$ vertices $\{1,2, \ldots, n\}$. Each vertex $i$ has the label $a_{i}$ and represents the position of the letter $a_{i}, 1 \leq i \leq n$, in $w$. For each occurrence of the subword $a b$ in $w$, there is an edge in $G(w)$ joining the vertices corresponding to the positions of $a$ and $b$ in $w$. We say that the graph $G$ is Parikh binary word representable by the binary word $w$. In other words, we say that a graph $G$ is Parikh binary word representable if there exists a binary word $w$ such that $G$ is Parikh binary word representable by the binary word $w$. 
Since every connected Parikh binary word representable graph corresponds to a core word, we deal with only core words and the corresponding graphs in the rest of this paper. As an illustration, if the core word is $w=a a b a b$, then in the Parikh word representable graph as shown in Figure 1, the vertices 1,2 and 4 have label $a$ while the vertices 3 and 5 have the label $b$. The number of edges in the graph is $|w|_{a b}=5$. For example there is a subword $a b$ in $w$ formed by the symbol $a$ in position 1 and the symbol $b$ in position 3 and so in the graph there is an edge joining the vertex 1 with the vertex 3 .

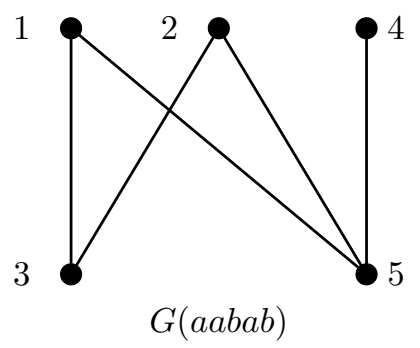

Figure 1: The Parikh word representable graph of the word $a a b a b$.

\section{Wiener index of Parikh word representable graphs}

Let $G=(V, E)$ be a connected graph with vertex set $V(G)$ and edge set $E(G)$. The distance between the vertices $u$ and $v$ of $G$ is denoted by $d(u, v)$ and is defined as the length of a shortest path between $u$ and $v$ in $G$.

Definition 3.1. The Wiener index $W(G)$ of a connected graph $G=(V, E)$, is the sum of distances $d(u, v)$ between all the vertices $u$ and $v$ of $G$. In other words

$$
W(G)=\sum_{\{u, v\} \subseteq V(G)} d(u, v) .
$$

We now obtain a formula for computing the Wiener index of Parikh word representable graph of a binary word.

Theorem 3.2. The Wiener index of a Parikh word representable graph $G(w)$ for $w=$ $a^{n_{1}} b a^{n_{2}} b \cdots a^{n_{l}} b, n_{1} \geq 1, n_{k} \geq 0$ for $2 \leq k \leq l$, is

$$
W(G(w))=\left(\sum_{i=1}^{l} n_{i}\right)^{2}+\sum_{i=1}^{l}(l+2 i-3) n_{i}+l(l-1) .
$$

Proof. In the Parikh word representable graph $G(w)$ corresponding to the word $w=$ $a^{n_{1}} b a^{n_{2}} b \cdots a^{n_{l}} b$, we consider pairs of vertices $(u, v)$, with $u, v \in\{1,2, \ldots, n\}$ where the label of $u$ appears before the label of $v$ in $w$. There are now four cases to be considered:

(i) $u$ and $v$ are both labeled $a$;

(ii) $u$ is labeled $a$ and $v$ is labeled $b$;

(iii) $u$ is labeled $b$ and $v$ is labeled $a$; 
(iv) $u$ and $v$ are both labeled $b$.

The contribution to the Wiener index of the Parikh word representable graph from each of these four cases may be calculated as follows:

(i) The distance between any two vertices labeled $a$ is 2 and there are $n_{1}+n_{2}+\cdots+n_{l}$ vertices labeled $a$. Hence the total contribution from these pairs of vertices is

$$
\left(n_{1}+n_{2}+\cdots+n_{l}\right) C_{2} \times 2=\left(n_{1}+n_{2}+\cdots+n_{l}\right)^{2}-\left(n_{1}+n_{2}+\cdots+n_{l}\right) .
$$

(ii) The distance between $u$ labeled $a$ and $v$ labeled $b$ is 1 and there are $n_{1}+\left(n_{1}+n_{2}\right)+$ $\cdots+\left(n_{1}+n_{2}+\cdots+n_{l}\right)$ such pairs. Hence the total contribution is

$$
n_{1}+\left(n_{1}+n_{2}\right)+\cdots+\left(n_{1}+n_{2}+\cdots+n_{l}\right)=\ln n_{1}+(l-1) n_{2}+\cdots+n_{l} .
$$

(iii) The distance between $u$ labeled $b$ and $v$ labeled $a$ is 3 and there are $\left(n_{2}+n_{3}+\cdots+\right.$ $\left.n_{l}\right)+\left(n_{3}+\cdots+n_{l}\right)+\cdots+n_{l}=n_{2}+2 n_{3}+\cdots+(l-1) n_{l}$ such pairs. Hence the total contribution is

$$
3\left(n_{2}+2 n_{3}+\cdots+(l-1) n_{l}\right)
$$

(iv) The distance between any two vertices labeled $b$ is 2 and there are $l$ vertices labeled $b$. Hence the total contribution from these pairs of vertices is

$$
l C_{2} \times 2=l(l-1) .
$$

Hence the Wiener index of $G(w)$ is given by

$$
\begin{aligned}
W(G(w))= & \left(n_{1}+n_{2}+\cdots+n_{l}\right)^{2}+(l-1) n_{1}+(l+1) n_{2}+\cdots \\
& +3(l-1) n_{l}+l(l-1) \\
= & \left(\sum_{i=1}^{l} n_{i}\right)^{2}+\sum_{i=1}^{l}(l+2 i-3) n_{i}+l(l-1) .
\end{aligned}
$$

Example 3.3. For the $P W R G$ in Figure 1 corresponding to the word $w=a^{2} b a b$, we have $l=2, n_{1}=2, n_{2}=1$ and so $W(G(w))=16$ which can also be verified from the formula in the Definition 3.1 by actually computing the distances $d(u, v)$ for all unordered pairs $(u, v)$ of vertices.

We now derive an alternate form of the expression for the Wiener index of the Parikh word representable graph $G(w)$ for $w=a^{n_{1}} b a^{n_{2}} b \cdots a^{n_{l}} b, n_{1} \geq 1, n_{k} \geq 0$ for $2 \leq k \leq l$. An interesting aspect of this alternate form is that the expression in the formula is elegant involving only the parameters related to the word.

Theorem 3.4. The Wiener index of a Parikh word representable graph $G(w)$, for $w=$ $a^{n_{1}} b a^{n_{2}} b \cdots a^{n_{l}} b, n_{1} \geq 1, n_{k} \geq 0$ for $2 \leq k \leq l$, is

$$
W(G(w))=|w|^{2}-|w|+|w|_{a}|w|_{b}-2|w|_{a b} .
$$


Proof. Since $w=a^{n_{1}} b a^{n_{2}} b \cdots a^{n_{l}} b$, we have $\sum_{i=1}^{l} n_{i}=|w|_{a}, l=|w|_{b}$. Also

$$
\begin{aligned}
|w|_{a}|w|_{b}-|w|_{a b} & =l\left(\sum_{i=1}^{l} n_{i}\right)-\left[\left(n_{1}+n_{2}+\cdots+n_{l}\right)+\cdots+\left(n_{1}+n_{2}\right)+n_{1}\right] \\
& =\sum_{i=1}^{l} i n_{i}-|w|_{a}
\end{aligned}
$$

so that

$$
\sum_{i=1}^{l} i n_{i}=|w|_{a}|w|_{b}+|w|_{a}-|w|_{a b} .
$$

Hence from Theorem 3.2, the Wiener index

$$
\begin{aligned}
W(G(w)) & =|w|_{a}^{2}+\left(|w|_{b}-3\right)|w|_{a}+|w|_{b}\left(|w|_{b}-1\right)+2 \sum_{i=1}^{l} i n_{i} \\
& =|w|_{a}^{2}+|w|_{b}^{2}+3|w|_{a}|w|_{b}-|w|_{a}-|w|_{b}-2|w|_{a b} \\
& =|w|^{2}-|w|+|w|_{a}|w|_{b}-2|w|_{a b}
\end{aligned}
$$

using $|w|=|w|_{a}+|w|_{b}$.

Corollary 3.5. The Wiener index of a Parikh word representable graph $G(w)$ for a fair word $w=a^{n_{1}} b a^{n_{2}} b \cdots a^{n_{l}} b, n_{1} \geq 1, n_{k} \geq 0$ for $2 \leq k \leq l$, is

$$
W(G(w))=|w|^{2}-|w|
$$

Proof. For a binary word $w$, we have $|w|_{a b}+|w|_{b a}=|w|_{a}|w|_{b}$. Since $w$ is a fair word, $|w|_{a b}=|w|_{b a}$ so that $|w|_{a}|w|_{b}-2|w|_{a b}=|w|_{b a}-|w|_{a b}=0$. Hence from Theorem 3.4, $W(G(w))=|w|^{2}-|w|$.

Theorem 3.6. The Wiener index $W(G(w))$ of a Parikh word representable graph $G(w)=$ $\left(V_{1} \cup V_{2}, E\right)$ with $\left|V_{1}\right|=|w|_{a}=p,\left|V_{2}\right|=|w|_{b}=q$ for the word $w=a^{n_{1}} b a^{n_{2}} b \cdots a^{n_{q}} b$, $n_{1} \geq 1, n_{k} \geq 0$ for $2 \leq k \leq l$, is bounded above by $p^{2}+q^{2}+3 p q-3 p-3 q+2$ and below by $p^{2}+q^{2}+p q-p-q$. The bounds are attained on $G\left(a b^{q-1} a^{p-1} b\right)$ and $G\left(a^{p} b^{q}\right)$ respectively.

Proof. Since $G(w)$ is connected, $|w|_{a b}=|E| \geq p+q-1$ [5]. Also $|w|_{a b} \leq p q$ [26]. Hence from Theorem 3.4, the Wiener index of $G(w)$ is

$$
\begin{aligned}
W(G(w)) & =p^{2}+q^{2}+3 p q-p-q-2|w|_{a b} \\
& \leq p^{2}+q^{2}+3 p q-p-q-2(p+q-1)=p^{2}+q^{2}+3 p q-3 p-3 q+2
\end{aligned}
$$

which is the Wiener index of the Parikh word representable graph $G\left(a b^{q-1} a^{p-1} b\right)$ and

$$
W(G(w)) \geq p^{2}+q^{2}+3 p q-p-q-2 p q=p^{2}+q^{2}+p q-p-q
$$

which is the Wiener index of the Parikh word representable graph $G\left(a^{p} b^{q}\right)$. 
Remark 3.7. One of the conjectures listed in [22, page 333], states that for a graph $G$ with diameter $d$ and order $2 d+1$, the Wiener index $W(G) \leq W\left(C_{2 d+1}\right)$ where $C_{2 d+1}$ denotes a cycle of length $2 d+1$. Since the diameter of any PWRG $G(w)$ corresponding to the binary core word $w$ is 3 , this conjecture holds good for $G(w)$, if the order of $G(w)$ is 7 , which also equals $|w|$. In fact, if the binary core word $w$ with $|w|=7$, is over the ordered alphabet $\{a<b\}$, the maximum Wiener index of $G(w)$ equals $W\left(C_{7}\right)$ which is 42 and this is attained, by Theorem 3.6, on $G\left(a b^{3} a^{2} b\right)$ or $G\left(a b^{2} a^{3} b\right)$.

We shall now find an expression for an upper bound on the Wiener index of Parikh word representable graph with a fixed number of edges. We use the following lemma.

Lemma 3.8. Given a fixed value of e and of $l$, the maximum value of $W(G(w))$ over all Parikh word representable graphs of the form $G(w)$ for $w=a^{n_{1}} b a^{n_{2}} b \cdots a^{n_{l}} b, n_{1} \geq 1$, $n_{k} \geq 0$ for $2 \leq k \leq l$, with e edges, is attained on $G\left(a b^{l-1} a^{e-l} b\right)$.

Proof. Since the number of edges in $G(w)$ is $e=|w|_{a b},|w|_{b}=l$ and $e \geq|w|_{a}+|w|_{b}-1$ as $G(w)$ is a connected graph with $|w|_{a}+|w|_{b}$ vertices, we have $|w|_{a} \leq e-l+1$. Hence from Theorem 3.4, the Wiener index of $G(w)$ is

$$
\begin{aligned}
W(G(w)) & =|w|_{a}\left(|w|_{a}-1\right)+l^{2}+3|w|_{a} l-l-2 e \\
& \leq(e-l+1)(e-l)+l^{2}+3|w|_{a} l-l-2 e \\
& \leq(e-l+1)(e-l)+l^{2}+3(e-l+1) l-l-2 e=e^{2}-l^{2}+e l+l-e
\end{aligned}
$$

which is the Wiener index of the Parikh word representable graph $G\left(a b^{l-1} a^{e-l} b\right)$.

Theorem 3.9. An upper bound of the Wiener index $W(G(w))$ of a Parikh word representable graph $G(w), w=a^{n_{1}} b a^{n_{2}} b \cdots a^{n_{l}} b, n_{1} \geq 1, n_{k} \geq 0$ for $2 \leq k \leq l$, with e edges is given by

$$
W(G(w)) \leq \begin{cases}5 m^{2}-6 m+2(m \geq 1), & \text { if } e=2 m-1 \\ 5 m^{2}-m(m \geq 1), & \text { if } e=2 m .\end{cases}
$$

The bound is sharp and is attained on $G\left(a b^{m-1} a^{m-1} b\right)$ when $e=2 m-1$ and on $G\left(a b^{m-1} a^{m} b\right)$ when $e=2 m$.

Proof. From Lemma 3.8, the Wiener index of the Parikh word representable graph $G(w)$, $w=a^{n_{1}} b a^{n_{2}} b \cdots a^{n_{l}} b, n_{1} \geq 1$, has a maximum for $G\left(a b^{l-1} a^{e-l} b\right)$ and is given by $W(G(w))=e^{2}-l^{2}+e l+l-e$. We now use the fact that a quadratic expression $a x^{2}+b x+c, a<0$, has a maximum when $x=-\frac{b}{2 a}$. When $e=2 m-1, m \geq 1$, we have

$$
W(G(w))=-l^{2}+2 m l+\left(4 m^{2}-6 m+2\right) .
$$

If $e=2 m-1$ has a fixed value, this quadratic expression in $l$ has a maximum when $l=m$ and the maximum is $5 m^{2}-6 m+2$. When $e=2 m, m \geq 1$, we have

$$
W(G(w))=-l^{2}+l(1+2 m)+\left(4 m^{2}-2 m\right) .
$$

Again if $e=2 m$ has a fixed value, this quadratic expression has a maximum when $l=$ $\left[m+\frac{1}{2}\right]=m$ where $[x]$ is the integral part of $x$ and the maximum is $5 m^{2}+2 m$.

We shall now evaluate the Wiener index of a Parikh word representable graph corresponding to a specific partition of a given integer. 
Theorem 3.10. Suppose $m_{1}+m_{2}+\cdots+m_{l}$ is a partition of some positive integer, where $m_{1} \geq m_{2} \geq \cdots \geq m_{l} \geq 1$. Then the Wiener index of the Parikh word representable graph $G$ corresponding to this partition is given by

$$
W(G)=m_{1}^{2}-2 e+(3 l-1) m_{1}+l(l-1)
$$

where $e$ is the number of edges of $G$.

Proof. From Lemma 2.4, the word $w=a^{m_{l}} b a^{m_{l-1}-m_{l}} b a^{m_{l-2}-m_{l-1}} b \cdots a^{m_{1}-m_{2}} b$ corresponds to the given partition. Now $|w|=m_{1}+l,|w|_{a}=m_{1},|w|_{b}=l$, so that using the formula in Theorem 3.4, we have

$$
\begin{aligned}
W(G) & =W(G(w))=|w|^{2}-|w|+|w|_{a}|w|_{b}-2|w|_{a b} \\
& =\left(m_{1}+l\right)^{2}-\left(m_{1}+l\right)+l m_{1}-2 e=m_{1}^{2}-2 e+(3 l-1) m_{1}+l(l-1)
\end{aligned}
$$

since $|w|_{a b}=e$.

\section{Multiplicative Wiener index}

Definition 4.1 ([32]). The Wiener polynomial of a graph $G$ is

$$
W(G ; x)=\sum_{\{u, v\} \subseteq V(G)} x^{d(u, v)}
$$

Theorem 4.2. The Wiener polynomial of a Parikh word representable graph $G(w)$, for $w=a^{n_{1}} b a^{n_{2}} b \cdots a^{n_{l}} b, n_{1} \geq 1, n_{k} \geq 0$ for $2 \leq k \leq l$, is

$W(G(w) ; x)=\left(|w|_{a}|w|_{b}-|w|_{a b}\right) x^{3}+\frac{1}{2}\left(|w|_{a}\left(|w|_{a}-1\right)+|w|_{b}\left(|w|_{b}-1\right)\right) x^{2}+\left(|w|_{a b}\right) x$

Proof. We consider pairs of vertices $(u, v)$ in the Parikh word representable graph $G(w)$ corresponding to the word $w=a^{n_{1}} b a^{n_{2}} b \cdots a^{n_{l}} b$, with $u, v \in\{1,2, \ldots, n\}$ where the label of $u$ appears before the label of $v$ in $w$. As discussed in the proof of Theorem 3.2, the vertex pairs are of four types, namely, types $1,2,3$ and 4 . Also, as discussed in the proof of Theorem 3.4 , there are $\frac{1}{2}|w|_{a}\left(|w|_{a}-1\right)$ pairs of vertices of type 1 and $\frac{1}{2}|w|_{b}\left(|w|_{b}-1\right)$ of type 4 and the distance between each such pair is 2 . Likewise, there are $|w|_{a b}$ pairs of vertices of type 2 with distance 1 and $|w|_{a}|w|_{b}-|w|_{a b}$ pairs of vertices of type 3 with distance 3 . Hence the Wiener polynomial is

$$
\left(|w|_{a}|w|_{b}-|w|_{a b}\right) x^{3}+\frac{1}{2}\left(|w|_{a}\left(|w|_{a}-1\right)+|w|_{b}\left(|w|_{b}-1\right)\right) x^{2}+\left(s|w|_{a b}\right) x .
$$

Definition 4.3 ([40]). The Wiener polarity index of $G$, denoted by $W_{p}(G)$, is defined as $W_{p}(G)=|\{(u, v) \subseteq V(G): d(u, v)=3\}|$.

Theorem 4.4. The Wiener polarity index of a Parikh word representable graph $G(w)$, for $w=a^{n_{1}} b a^{n_{2}} b \cdots a^{n_{l}} b, n_{1} \geq 1, n_{k} \geq 0$ for $2 \leq k \leq l$, is given by

$$
W_{p}(G(w))=|w|_{a}|w|_{b}-|w|_{a b}
$$


Proof. In the Parikh word representable graph $G(w)$, for the word $w=a^{n_{1}} b a^{n_{2}} b \cdots a^{n_{l}} b$ as in the hypothesis, the pairs of vertices $(u, v)$ of type 3 as mentioned in the proof of Theorem 4.2, are at a distance 3 and these pairs contribute to the Wiener polarity index of $G(w)$. In fact there are $n_{2}+n_{3}+\cdots+n_{l}$ vertices with label $a$ that are at distance 3 from the vertex with label, the first $b$ in $w$. Likewise for other vertices corresponding to the other $b^{\prime} s$ in $w$. Hence

$$
\begin{aligned}
W_{p}(G(w)) & =\left(n_{2}+n_{3}+\cdots+n_{l}\right)+\left(n_{3}+n_{4}+\cdots+n_{l}\right)+\cdots+n_{l} \\
& =\sum_{i=1}^{l} i n_{i}-\sum_{i=1}^{l} n_{i}=\left.w\right|_{a}|w|_{b}-|w|_{a b} .
\end{aligned}
$$

Definition 4.5 ([13]). The multiplicative version of the Wiener index of a graph $G$ is

$$
\pi(G)=\prod_{\{u, v\} \subseteq V(G)} d(u, v) .
$$

Theorem 4.6. The multiplicative Wiener index of a Parikh word representable graph $G(w)$, for $w=a^{n_{1}} b a^{n_{2}} b \cdots a^{n_{l}} b, n_{1} \geq 1, n_{k} \geq 0$ for $2 \leq k \leq l$, is

$$
\pi(G(w))=2^{\frac{|w|_{a}\left(|w|_{a}-1\right)+|w|_{b}\left(|w|_{b}-1\right)}{2}} 3^{|w|_{a}|w|_{b}-|w|_{a b}} .
$$

Proof. Considering pairs of vertices as in Theorem 4.2, we obtain the required result, since there are $\frac{|w|_{a}\left(|w|_{a}-1\right)+|w|_{b}\left(|w|_{b}-1\right)}{2}$ pairs of vertices at distance 2 in $G(w)$ while there are $|w|_{a}|w|_{b}-|w|_{a b}$ pairs of vertices at distance 3 . It is to be noted that pairs of vertices at distance 1 contribute value 1 to the product defining $\pi(G(w))$.

Corollary 4.7. The multiplicative Wiener index of a Parikh word representable graph $G(w)$ for a fair word $w=a^{n_{1}} b a^{n_{2}} b \cdots a^{n_{l}} b, n_{1} \geq 1, n_{k} \geq 0$ for $2 \leq k \leq l$, is

$$
\pi(G(w))=2^{\frac{|w|^{2}-|w|}{2}}\left(\frac{3}{2}\right)^{|w|_{a b}}
$$

Proof. For a binary word $w$, we have $|w|_{a b}+|w|_{b a}=|w|_{a}|w|_{b}$. Since $w$ is a fair word, $|w|_{a b}=|w|_{b a}$ so that $|w|_{a}|w|_{b}-2|w|_{a b}=|w|_{b a}-|w|_{a b}=0$. Also $|w|=|w|_{a}+|w|_{b}$. Hence from Theorem 4.6, $\pi(G(w))=2^{\frac{|w|^{2}-|w|}{2}}\left(\frac{3}{2}\right)^{|w|_{a b}}$.

Lemma 4.8. Given a fixed value of e and of $l$, the maximum value of $\pi(G(w))$ over all Parikh word representable graphs of the form $G(w)$ for $w=a^{n_{1}} b a^{n_{2}} b \cdots a^{n_{l}} b, n_{1} \geq 1$, $n_{k} \geq 0$ for $2 \leq k \leq l$, with e edges, is attained on $G\left(a b^{l-1} a^{e-l} b\right)$.

Proof. Since $|w|_{b}=l$ and the number of edges in $G(w)$ is $e=|w|_{a b}$, we have $|w|_{a} \leq$ $e-l+1$ as $G(w)$ is a connected graph with $|w|_{a}+|w|_{b}$ vertices so that $e \geq|w|_{a}+|w|_{b}-1$. Note that in a connected graph $G$ with $n$ vertices and $e$ edges, $e \geq n-1$ [5]. Hence from Theorem 4.6, the multiplicative Wiener index of $G(w)$ is

$$
\begin{aligned}
\pi(G(w)) & =2^{\frac{|w|_{a}\left(|w|_{a}-1\right)+|w|_{b}\left(|w|_{b}-1\right)}{2}} 3^{|w|_{a}|w|_{b}-|w|_{a b}} \\
& \leq 2^{\frac{(e-l+1)(e-l)+l(l-1)}{2}} 3^{(e-l+1) l-e}
\end{aligned}
$$

which is the multiplicative Wiener index of the Parikh word representable graph $G\left(a b^{l-1} a^{e-l} b\right)$. 
Theorem 4.9. An upper bound of the multiplicative Wiener index $\pi(G(w))$ of a Parikh word representable graph $G(w), w=a^{n_{1}} b a^{n_{2}} b \cdots a^{n_{l}} b, n_{1} \geq 1, n_{k} \geq 0$ for $2 \leq k \leq l$, with e edges is given by

$$
\pi(G(w)) \leq \begin{cases}2^{m^{2}-m} 3^{m^{2}-2 m+1}(m \geq 1), & \text { if } e=2 m-1 \\ 2^{m^{2}} 3^{m^{2}-m}(m \geq 1), & \text { if } e=2 m\end{cases}
$$

The bound is sharp and is attained on $G\left(a b^{m-1} a^{m-1} b\right)$ when $e=2 m-1$ and on $G\left(a b^{m-1} a^{m} b\right)$ when $e=2 m$.

Proof. From Lemma 4.8, the multiplicative Wiener index of the Parikh word representable graph $G(w), w=a^{n_{1}} b a^{n_{2}} b \cdots a^{n_{l}} b, n_{1} \geq 1$, has a maximum for $G\left(a b^{l-1} a^{e-l} b\right)$ and is given by

$$
\pi(G(w))=2^{\frac{(e-l+1)(e-l)+l(l-1)}{2}} 3^{(e-l+1) l-e} .
$$

On taking logarithms we get,

$$
\begin{aligned}
\ln (\pi(G(w))) & =\left[(e-l+1)(e-l)+l^{2}-l\right] \frac{\ln 2}{2}+[(e-l+1) l-e] \ln 3 \\
& =l^{2}(\ln 2-\ln 3)-l\left((e+1)(\ln 2-\ln 3)+e\left(\frac{(e+1)}{2} \ln 2-\ln 3\right) .\right.
\end{aligned}
$$

We now use the fact that a quadratic expression $a x^{2}+b x+c, a<0$, has a maximum when $x=-\frac{b}{2 a}$. Let $F(l)=l^{2}(\ln 2-\ln 3)-l\left((e+1)(\ln 2-\ln 3)+e\left(\frac{(e+1)}{2} \ln 2-\ln 3\right)\right.$. When $e=2 m-1, m \geq 1, F(l)$ has a maximum when $l=m$ and so $\pi(G(w))$ has the maximum $2^{m^{2}-m} 3^{m^{2}-2 m+1}$. When $e=2 m, m \geq 1, F(l)$ has a maximum when $l=\left[m+\frac{1}{2}\right]=m$ where $[x]$ is the integral part of $x$ and $\pi(G(w))$ has the maximum $2^{m^{2}} 3^{m^{2}-m}$.

Theorem 4.10. The multiplicative Wiener index $\pi(G(w))$ of a Parikh word representable graph $G(w)=\left(V_{1} \cup V_{2}, E\right)$ with $\left|V_{1}\right|=|w|_{a}=p,\left|V_{2}\right|=|w|_{b}=q$ for the word $w=a^{n_{1}} b a^{n_{2}} b \cdots a^{n_{q}} b, n_{1} \geq 1, n_{k} \geq 0$ for $2 \leq k \leq l$, is bounded above by

$$
2^{\frac{p(p-1)+q(q-1)}{2}} 3^{p q-p-q+1}
$$

and below by

$$
2^{\frac{p(p-1)+q(q-1)}{2}} .
$$

The bounds are attained on $G\left(a b^{q-1} a^{p-1} b\right)$ and $G\left(a^{p} b^{q}\right)$ respectively.

Proof. Since $G(w)$ is connected, $|w|_{a b}=|E| \geq p+q-1$ [5]. Also $|w|_{a b} \leq p q$ [26]. Hence from Theorem 4.6, the multiplicative Wiener index of $G(w)$ is

$$
\pi(G(w)) \geq 2^{\frac{p(p-1)+q(q-1)}{2}}
$$

which is the multiplicative Wiener index of the Parikh word representable graph $G\left(a^{p} b^{q}\right)$ and

$$
\pi(G(w)) \leq 2^{\frac{p(p-1)+q(q-1)}{2}} 3^{p q-p-q+1}
$$

which is the multiplicative Wiener index of the Parikh word representable graph $G\left(a b^{q-1} a^{p-1} b\right)$. 


\section{Hyper-Wiener index of Parikh word representable graphs}

We now derive formuas for computing hyper-Wiener index of Parikh word representable graphs of binary words.

Definition 5.1 ([20, 30]). The hyper-Wiener index of a connected graph $G$ is given by

$$
W W(G)=\sum_{\{u, v\} \subseteq V(G)} d(u, v)+d^{2}(u, v)
$$

where $d(u, v)$ is the distance between the vertices $u$ and $v$ of $G$.

Theorem 5.2. The hyper-Wiener index $W W(G(w))$ of a Parikh word representable graph $G(w)$ for $w=a^{n_{1}} b a^{n_{2}} b \cdots a^{n_{l}} b, n_{1} \geq 1, n_{k} \geq 0$ for $2 \leq k \leq l$, is

$$
W W(G(w))=3\left(\sum_{i=1}^{l} n_{i}\right)^{2}+\sum_{i=1}^{l}(2 l+10 i-13) n_{i}+3 l(l-1) .
$$

Proof. As in the proof of Theorem 3.2, there are four cases for the vertex pairs $(u, v)$. The contribution to the hyper-Wiener index of the Parikh word representable graph from each of these cases may be calculated as follows:

(i) The contribution from pairs of vertices labeled $a$ is

$$
\left(n_{1}+n_{2}+\cdots+n_{l}\right) C_{2} \times(2+4)=3\left(n_{1}+n_{2}+\ldots+n_{l}\right)^{2}-3\left(n_{1}+n_{2}+\cdots+n_{l}\right) .
$$

(ii) The contribution from pairs of vertices $(u, v)$ where $u$ is labeled $a$ and $v$ is labeled $b$ is

$$
2\left(n_{1}+\left(n_{1}+n_{2}\right)+\ldots+\left(n_{1}+n_{2}+\ldots+n_{l}\right)\right)=2\left(\ln _{1}+(l-1) n_{2}+\cdots+n_{l}\right) .
$$

(iii) The contribution from pairs of vertices $(u, v)$ where $u$ is labeled $b$ and $v$ is labeled $a$ is

$$
\left.12\left(n_{2}+2 n_{3}+\cdots+(l-1) n_{l}\right)\right) .
$$

(iv) The contribution from pairs of vertices labeled $b$ is

$$
l C_{2} \times 6=3 l(l-1) .
$$

Hence the hyper-Wiener index of $G(w)$ is given by

$$
W W\left(G(w)=3\left(\sum_{i=1}^{l} n_{i}\right)^{2}+\sum_{i=1}^{l}(2 l+10 i-13) n_{i}+3 l(l-1) .\right.
$$

We now derive an alternate form of the expression for the hyper-Wiener index of the Parikh word representable graph $G(w)$ for $w=a^{n_{1}} b a^{n_{2}} b \cdots a^{n_{l}} b, n_{1} \geq 1, n_{k} \geq 0$ for $2 \leq k \leq l$. 
Theorem 5.3. The hyper-Wiener index of a Parikh word representable graph $G(w)$ for $w=a^{n_{1}} b a^{n_{2}} b \cdots a^{n_{l}} b, n_{1} \geq 1, n_{k} \geq 0$ for $2 \leq k \leq l$, is

$$
\begin{aligned}
W W(G(w)) & =3|w|_{a}^{2}+3|w|_{b}^{2}+12|w|_{a}|w|_{b}-3|w|_{a}-3|w|_{b}-10|w|_{a b} \\
& =3|w|^{2}-3|w|+6|w|_{a}|w|_{b}-10|w|_{a b} .
\end{aligned}
$$

Proof. Since $w=a^{n_{1}} b a^{n_{2}} b \cdots a^{n_{l}} b$, we have $\sum_{i=1}^{l} n_{i}=|w|_{a}, l=|w|_{b}$. Also, as in the proof of Theorem 3.4,

$$
\sum_{i=1}^{l} i n_{i}=|w|_{a}|w|_{b}+|w|_{a}-|w|_{a b} .
$$

Hence from Theorem 5.2, the hyper-Wiener index

$$
\begin{aligned}
W W(G(w)) & =3|w|_{a}^{2}+\left(2|w|_{b}-13\right)|w|_{a}+3|w|_{b}\left(|w|_{b}-1\right)+10 \sum_{i=1}^{l} i n_{i} \\
& =3|w|_{a}^{2}+3|w|_{b}^{2}+12|w|_{a}|w|_{b}-3|w|_{a}-3|w|_{b}-10|w|_{a b} \\
& =3|w|^{2}-3|w|+6|w|_{a}|w|_{b}-10|w|_{a b}
\end{aligned}
$$

using $|w|=|w|_{a}+|w|_{b}$.

Theorem 5.4. The hyper-Wiener index of a Parikh word representable graph $G(w)=$ $\left(V_{1} \cup V_{2}, E\right)$ with $\left|V_{1}\right|=|w|_{a}=p,\left|V_{2}\right|=|w|_{b}=q$ for the word $w=a^{n_{1}} b a^{n_{2}} b \cdots a^{n_{q}} b$, $n_{1} \geq 1, n_{k} \geq 0$ for $2 \leq k \leq l$, is bounded above and below by

$$
3\left(p^{2}+q^{2}\right)+12 p q-13 p-13 q+10
$$

and

$$
3\left(p^{2}+q^{2}\right)+2 p q-3 p-3 q .
$$

The bounds are attained on $G\left(a b^{q-1} a^{p-1} b\right)$ and $G\left(a^{p} b^{q}\right)$ respectively.

Proof. As done in the proof of Theorem 3.6, using the inequalities $|w|_{a b} \geq p+q-1$ [5], $|w|_{a b} \leq p q$ [26], we have from Theorem 5.3, the hyper-Wiener index of $G(w)$ is

$$
\begin{aligned}
W W(G(w)) & =3 p^{2}+3 q^{2}+12 p q-3 p-3 q-10|w|_{a b} \\
& \leq 3\left(p^{2}+q^{2}\right)+12 p q-13 p-13 q+10
\end{aligned}
$$

which is the hyper-Wiener index of the Parikh word representable graph $G\left(a b^{q-1} a^{p-1} b\right)$ and

$$
W(G(w)) \geq 3\left(p^{2}+q^{2}\right)+2 p q-3 p-3 q
$$

which is the Wiener index of the Parikh word representable graph $G\left(a^{p} b^{q}\right)$.

The hyper-Wiener index of a Parikh word representable graph corresponding to a specific partition of a given integer can be evaluated proceeding as in the proof of Theorem 3.10 and is given in the following theorem. 
Theorem 5.5. Suppose $m_{1}+m_{2}+\cdots+m_{l}$ is a partition of some positive integer, where $m_{1} \geq m_{2} \geq \cdots \geq m_{l} \geq 1$. Then the hyper-Wiener index of the Parikh word representable graph $G$ corresponding to this partition is given by

$$
W W(G)=3\left(m_{1}^{2}+(4 l-1) m_{1}+l(l-1)\right)-10 e
$$

where $e$ is the number of edges of $G$.

We shall now find an expression for an upper bound on the hyper-Wiener index of Parikh word representable graph with a fixed number of edges. We use the following lemma.

Lemma 5.6. Given a fixed value of e and of $l$, the maximum value of $W W(G(w))$ over all Parikh word representable graphs of the form $G(w)$ for $w=a^{n_{1}} b a^{n_{2}} b \cdots a^{n_{l}} b, n_{1} \geq 1$, $n_{k} \geq 0$ for $2 \leq k \leq l$, with e edges, is attained on $G\left(a b^{l-1} a^{e-l} b\right)$.

Proof. Proceeding as done in the proof of Lemma 3.8, the number of edges in $G(w)$ is $e=|w|_{a b},|w|_{b}=l$ and $e \geq|w|_{a}+|w|_{b}-1$ as $G(w)$ is a connected graph with $|w|_{a}+|w|_{b}$ vertices, we have $|w|_{a} \leq e-l+1$. Hence from Theorem 5.3, the hyper-Wiener index of $G(w)$ is

$$
\begin{aligned}
W W(G(w)) & =3|w|_{a}\left(|w|_{a}-1\right)+3 l^{2}+12 l|w|_{a}-3 l-10 e \\
& \leq 3(e-l+1)(e-l)+3 l^{2}+12 l(e-l+1)-3 l-10 e \\
& =3 e^{2}-6 l^{2}+6 e l+6 l-7 e
\end{aligned}
$$

which is the hyper-Wiener index of the Parikh word representable graph $G\left(a b^{l-1} a^{e-l} b\right)$.

Theorem 5.7. An upper bound of the Wiener index $W(G(w))$ of a Parikh word representable graph $G(w)$ for $w=a^{n_{1}} b a^{n_{2}} b \cdots a^{n_{l}} b, n_{1} \geq 1, n_{k} \geq 0$ for $2 \leq k \leq l$, with e edges is given by

$$
W W(G) \leq \begin{cases}18 m^{2}-26 m+10(m \geq 1), & \text { if } e=2 m-1 \\ 18 m^{2}-8 m(m \geq 1), & \text { if } e=2 m\end{cases}
$$

The bound is sharp and is attained on $G\left(a b^{m-1} a^{m-1} b\right)$ when $e=2 m-1$ and on $G\left(a b^{m-1} a^{m} b\right)$ when $e=2 m$.

Proof. From Lemma 5.6, the hyper-Wiener index of the Parikh word representable graph $G(w), w=a^{n_{1}} b a^{n_{2}} b \cdots a^{n_{l}} b, n_{1} \geq 1$, has a maximum for $G(w)$ for $w=a b^{l-1} a^{e-l} b$ and is given by $W W(G(w))=3 e^{2}-6 l^{2}+6 e l+6 l-7 e$. We use the fact that a quadratic expression $a x^{2}+b x+c, a<0$, has a maximum when $x=-\frac{b}{2 a}$. When $e=2 m-1, m \geq 1$, we have $W W(G(w))=-6 l^{2}+12 m l+\left(12 m^{2}-26 m+10\right)$. If $e=2 m-1$ has a fixed value, this quadratic expression in $l$ has a maximum when $l=m$ and the maximum is $18 m^{2}-26 m+10$. When $e=2 m, m \geq 1$, we have $W W(G(w))=-6 l^{2}+l(6+$ $12 m)+\left(12 m^{2}-14 m\right)$. Again if $e=2 m$ has a fixed value, this quadratic expression has a maximum when $l=\left[m+\frac{1}{2}\right]=m$ where $[x]$ is the integral part of $x$ and the maximum is $18 m^{2}-8 m$. 


\section{Terminal and peripheral Wiener indices}

By considering only pendant vertices in a graph, a special kind of Wiener index, called terminal Wiener index, has been introduced and studied [11]. Here we consider this notion in the context of Parikh word representable graphs.

Definition 6.1 ([11]). The terminal Wiener index $T W(G)$ of a connected graph $G$ is the sum of distances between all pairs of pendant vertices of $G$.

Theorem 6.2. The terminal Wiener index of a Parikh word representable graph $G=$ $G\left(a^{n_{1}} b a^{n_{2}} b \cdots a^{n_{l}} b\right), n_{1} \geq 1, n_{k} \geq 0$ for $2 \leq k \leq l$, is given by

$$
T W(G)= \begin{cases}n_{l}\left(n_{l}-1\right), & \text { if } n_{1} \neq 1 ; \\ n_{l}\left(n_{l}-1\right)+k(k-1)+3 k n_{l}, & \text { if } n_{1}=1, \text { for } 2 \leq j \leq k \leq l, n_{j}=0 .\end{cases}
$$

Proof. If $n_{1} \neq 1$, the only possible pendant vertices are the $a$ 's in the last block and the distance between any two of them is 2 so that

$$
T W(G)=2 \times n_{l} C_{2}=n_{l}\left(n_{l}-1\right) .
$$

Note that if $n_{l}=0$, then $T W(G)=0$.

If $n_{1}=1, n_{2}=n_{3}=\cdots=n_{k}=0$ and $n_{k+1} \neq 0,2 \leq k<l, l>1$, then the first $k b$ 's are also a pendant vertices and the distance between any one of these $b$ 's and any one of the $a$ 's in the last block is 3 while that between any two $b$ 's is 2 as well as the distance between any two $a^{\prime} s$ in the last block is 2 . Hence

$$
T W(G)=n_{l}\left(n_{l}-1\right)+k(k-1)+3 k n_{l} .
$$

When $k=l$, then $n_{1}=1$ and $n_{i}=0,2 \leq i \leq l$ so that all the $b^{\prime} s$ are the only pendant vertices and hence

$$
T W(G)=l(l-1) .
$$

If $n_{1}=1, l=1$, clearly $T W(G)=1$ as the corresponding graph $G(a b)$ has only one edge with the end labels $a$ and $b$.

Another special kind of Wiener index, called peripheral Wiener index, has also been studied [16].

Definition 6.3 ([16]). The peripheral Wiener index of a connected graph $G$ is given by $W_{p}(G)=\sum_{\{u, v\} \subseteq P(G)} d(u, v)$ where $P(G)$ is the set of all peripheral vertices which are the vertices of $G$ with their eccentricities equal to diameter of $G$.

Theorem 6.4. The peripheral Wiener index of the Parikh word representable graph $G(w)$ for $w=a^{n_{1}} b a^{n_{2}} b a^{n_{3}} b \cdots a^{n_{r}} b^{l-r+1}, n_{1} \geq 1, n_{k} \geq 0$ for $2 \leq k \leq r-1$, and $n_{r}>0$ with $r$ at least 2 and at the most $l$, is

$$
P W(G(w))=\left(\sum_{i=2}^{r} n_{i}\right)^{2}+\sum_{i=2}^{r}(r+2 i-4) n_{i}+(r-1)(r-2) .
$$

Furthermore $P W(G(w))=W(G(w))$ if $w=a^{n} b^{l}$, where $W(G(w))$ is the Wiener index of $G(w)$. 
Proof. From the definition of $G(w)$, the Parikh word representable graph corresponding to the word $w=a^{n_{1}} b a^{n_{2}} b a^{n_{3}} b \cdots a^{n_{r}} b^{l-r+1}$, it is clear that $G(w)$ is a bipartite graph of diameter 3 and radius 2 . Also,the vertices corresponding to all $a^{\prime} s$ in $a^{n_{i}}, 2 \leq i \leq r$ and to all $b^{\prime} s$ except the last $l-r+1 b^{\prime} s$ are of eccentricity 3 and hence they are the peripheral vertices of $G(w)$. We consider pairs of peripheral vertices $(u, v)$, where the label of $u$ appears before the label of $v$ in $w$. As in Theorem 3.2, the vertex pairs of $(u, v)$ can be divided into four cases as given below:

1. $u$ and $v$ are both labeled $a$;

2. $u$ is labeled $a$ and $v$ is labeled $b$;

3. $u$ is labeled $b$ and $v$ is labeled $a$;

4. $u$ and $v$ are both labeled $b$.

The contribution to the peripheral Wiener index of the Parikh word representable graph from each of these cases may be calculated as follows:

1. The contribution from the pairs of peripheral vertices labeled $a$ is

$$
\left(n_{2}+n_{3}+n_{4}+\cdots+n_{r}\right) C_{2} \times 2=\left(n_{2}+n_{3}+n_{4}+\cdots+n_{r}\right)^{2}-\left(n_{2}+n_{3}+n_{4}+\cdots+n_{r}\right) .
$$

2. The contribution from the pairs of peripheral vertices $(u, v)$ where $u$ is labeled $a$ and $v$ is labeled $b$ is

$$
\begin{aligned}
n_{2}+\left(n_{2}+n_{3}\right)+\left(n_{2}+n_{3}+n_{4}\right) & +\cdots+\left(n_{2}+n_{3}+\cdots+n_{r-1}\right) \\
& =(r-2) n_{2}+(r-3) n_{3}+\cdots+n_{r-1} .
\end{aligned}
$$

3. The contribution from the pairs of peripheral vertices $(u, v)$ where $u$ is labeled $b$ and $v$ is labeled $a$ is

$$
\begin{aligned}
3\left[\left(n_{2}+n_{3}+\cdots+n_{r}\right)+\left(n_{3}+n_{4}\right.\right. & \left.\left.+\cdots+n_{r}\right)+\cdots+n_{r}\right] \\
& =3\left[(r-1) n_{r}+(r-2) n_{r-1}+\cdots+n_{2}\right] .
\end{aligned}
$$

4. The contribution from the pairs of peripheral vertices labeled $b$ is

$$
(r-1) C_{2} \times 2=(r-1)(r-2) .
$$

Hence the peripheral Wiener index of $G(w)$ is given by

$$
P W(G(w))=\left(\sum_{i=2}^{r} n_{i}\right)^{2}+\sum_{i=2}^{r}(r+2 i-4) n_{i}+(r-1)(r-2) .
$$

Furthermore, if $w=a^{n} b^{l}$ then all the vertices of $G(w)$ labeled $a$ as well as $b$ are peripheral vertices of $G(w)$. Thus $P W(G(w))=W(G(w))$ where $W(G(w))$ is the Wiener index of $G(w)$. 


\section{Conclusion}

We have derived formulas for evaluating the Wiener index and certain other variants of Wiener topological indices for Parikh word representable graphs [3] of binary core words. There are problems that remain to be investigated. For example, the lower bound of Wiener index of a Parikh word representable graph $G(w)$ of a binary core word $w$ when the number of edges of $G(w)$ is a given fixed value, needs to be examined. Bipartite graphs have been utilized in studies of structural features in the areas of molecular biology and chemistry (see, for example, $[17,35])$. Parikh word representable graphs $(P W R G)$ corresponding to binary core words are bipartite graphs. It will be of interest to examine the role of $P W R G$ in such studies of structural features and relationships. Also, it will also be of interest to study other kinds of topological indices for this class of graphs.

\section{ORCID iDs}

Nobin Thomas iD https://orcid.org/0000-0002-4057-3220

Lisa Mathew (D) https://orcid.org/0000-0002-3722-3326

Sastha Sriram (D) https://orcid.org/0000-0003-0604-2159

K. G. Subramanian (D) https://orcid.org/0000-0001-8726-5850

\section{References}

[1] A. Atanasiu, Binary amiable words, Internat. J. Found. Comput. Sci. 18 (2007), 387-400, doi: $10.1142 / \mathrm{s} 0129054107004735$.

[2] A. Atanasiu, R. Atanasiu and I. Petre, Parikh matrices and amiable words, Theoret. Comput. Sci. 390 (2008), 102-109, doi:10.1016/j.tcs.2007.10.022.

[3] S. Bera and K. Mahalingam, Structural properties of word representable graphs, Math. Comput. Sci. 10 (2016), 209-222, doi:10.1007/s11786-016-0257-1.

[4] S. Bera, K. Mahalingam and K. G. Subramanian, Properties of Parikh matrices of binary words obtained by an extension of a restricted shuffle operator, Internat. J. Found. Comput. Sci. 29 (2018), 403-413, doi:10.1142/s0129054118500119.

[5] G. Bondy and U. Murty, Graph Theory with Applications, North-Hollans, 1982.

[6] A. Černý, On fair words, J. Autom. Lang. Comb. 14 (2009), 163-174, doi:10.25596/ jalc-2009-163.

[7] A. Collins, S. Kitaev and V. V. Lozin, New results on word-representable graphs, Discrete Appl. Math. 216 (2017), 136-141, doi:10.1016/j.dam.2014.10.024.

[8] M. V. Diudea, Basic Chemical Graph Theory, in: Multi-shell Polyhedral Clusters, Springer, Cham, volume 10 of Carbon Materials: Chemistry and Physics, 2018 pp. 1-21, doi:10.1007/ 978-3-319-64123-2_1.

[9] M. Eliasi, G. Raeisi and B. Taeri, Wiener index of some graph operations, Discrete Appl. Math. 160 (2012), 1333-1344, doi:10.1016/j.dam.2012.01.014.

[10] A. L. L. Gao, S. Kitaev and P. B. Zhang, On 132-representable graphs, Australas. J. Combin. 69 (2017), 105-118, https://ajc.maths.uq.edu.au/pdf/69/ajc_v69_p105. pdf.

[11] I. Gutman, B. Furtula and M. Petrović, Terminal Wiener index, J. Math. Chem. 46 (2009), 522-531, doi:10.1007/s10910-008-9476-2. 
[12] I. Gutman, S. Li and W. Wei, Cacti with $n$-vertices and $t$ cycles having extremal Wiener index, Discrete Appl. Math. 232 (2017), 189-200, doi:10.1016/j.dam.2017.07.023.

[13] I. Gutman, W. Linert, I. Lukovits and Ž. Tomović, The multiplicative version of the Wiener index, J. Chem. Inf. Comput. Sci. 40 (2000), 113-116, doi:10.1021/ci990060s.

[14] I. Gutman and O. E. Polansky, Mathematical Concepts in Organic Chemistry, Springer-Verlag, Berlin, 1986, doi:10.1007/978-3-642-70982-1.

[15] M. M. Halldórsson, S. Kitaev and A. Pyatkin, Graphs capturing alternations in words, in: Y. Gao, H. Lu, S. Seki and S. Yu (eds.), Developments in Language Theory, Springer, Berlin, Heidelberg, volume 6224 of Lecture Notes in Computer Science, 2010 pp. 436-437, doi: 10.1007/978-3-642-14455-4_41, proceedings of the 14th International Conference (DLT 2010) held at the University of Western Ontario, London, ON, August 17 - 20, 2010.

[16] H. Hua, On the peripheral Wiener index of graphs, Discrete Appl. Math. 258 (2019), 135-142, doi:10.1016/j.dam.2018.11.031.

[17] P. Iyer and J. Bajorath, Mechanism-based bipartite matching molecular series graphs to identify structural modifications of receptor ligands that lead to mechanism hopping, Med. Chem. Commun. 3 (2012), 441-448, doi:10.1039/c2md00281g.

[18] S. Kitaev and V. Lozin, Words and Graphs, Monographs in Theoretical Computer Science, An EATCS Series, Springer, Cham, 2015, doi:10.1007/978-3-319-25859-1.

[19] S. Kitaev, P. Salimov, C. Severs and H. Ulfarsson, Word-representability of line graphs, Open J. Discrete Math. 1 (2011), 96-101, doi:10.4236/ojdm.2011.12012.

[20] D. J. Klein, I. Lukovits and I. Gutman, On the definition of the hyper-Wiener index for cyclecontaining structures, J. Chem. Inf. Comput. Sci. 35 (1995), 50-52, doi:10.1021/ci00023a007.

[21] M. Knor and R. Škrekovski, Wiener index of generalized 4-stars and of their quadratic line graphs, Australas. J. Combin. 58 (2014), 119-126, https: / / j jc.maths.uq. edu .au/ pdf/58/ajc_v58_p119.pdf.

[22] M. Knor, R. Škrekovski and A. Tepeh, Mathematical aspects of Wiener index, Ars Math. Contemp. 11 (2016), 327-352, doi:10.26493/1855-3974.795.ebf.

[23] M. Lothaire, Combinatorics on Words, Cambridge Mathematical Library, Cambridge University Press, Cambridge, 1997, doi:10.1017/cbo9780511566097.

[24] Y. Mandelshtam, On graphs representable by pattern-avoiding words, Discuss. Math. Graph Theory 39 (2019), 375-389, doi:10.7151/dmgt.2128.

[25] A. Mateescu and A. Salomaa, Matrix indicators for subword occurrences and ambiguity, Internat. J. Found. Comput. Sci. 15 (2004), 277-292, doi:10.1142/s0129054104002418.

[26] A. Mateescu, A. Salomaa, K. Salomaa and S. Yu, A sharpening of the Parikh mapping, Theor. Inform. Appl. 35 (2001), 551-564, doi:10.1051/ita:2001131.

[27] L. Mathew, N. Thomas, S. Bera and K. G. Subramanian, Some results on Parikh word representable graphs and partitions, Adv. Appl. Math. 107 (2019), 102-115, doi:10.1016/j.aam.2019. 02.009 .

[28] K. Pattabiraman and P. Paulraja, Wiener index of the tensor product of a path and a cycle, Discuss. Math. Graph Theory 31 (2011), 737-751, doi:10.7151/dmgt.1576.

[29] K. Pattabiraman and P. Paulraja, Wiener and vertex PI indices of the strong product of graphs, Discuss. Math. Graph Theory 32 (2012), 749-769, doi:10.7151/dmgt.1647.

[30] M. Randić, Novel molecular descriptor for structure-property studies, Chem. Phys. Lett. 211 (1993), 478-483, doi:10.1016/0009-2614(93)87094-j. 
[31] G. Rozenberg and A. Salomaa (eds.), Handbook of Formal Languages, Volume 1: Word, Language, Grammar, Springer-Verlag, Berlin, 1997, doi:10.1007/978-3-642-59136-5.

[32] B. E. Sagan, Y.-N. Yeh and P. Zhang, The Wiener polynomial of a graph, Int. J. Quantum Chem.

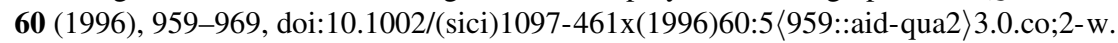

[33] A. Salomaa, Parikh matrices: subword indicators and degrees of ambiguity, in: H.-J. Böckenhauer, D. Komm and W. Unger (eds.), Adventures Between Lower Bounds and Higher Altitudes, Springer, Cham, volume 11011 of Lecture Notes in Computer Science, pp. 100-112, 2018, doi:10.1007/978-3-319-98355-4_7.

[34] K. G. Subramanian, A. M. Huey and A. K. Nagar, On Parikh matrices, Internat. J. Found. Comput. Sci. 20 (2009), 211-219, doi:10.1142/s0129054109006528.

[35] W. R. Taylor, Protein structure comparison using bipartite graph matching and its application to protein structure classification, Mol. Cell. Proteom. 1 (2002), 334-339, doi:10.1074/mcp. t200001-mcp200.

[36] W. C. Teh, On core words and the Parikh matrix mapping, Internat. J. Found. Comput. Sci. 26 (2015), 123-142, doi:10.1142/s0129054115500069.

[37] W. C. Teh, Parikh-friendly permutations and uniformly Parikh-friendly words, Australas. J. Combin. 76 (2020), 208-219, https: / / a jc.maths.uq.edu.au/pdf/76/ajc_v76_ p208.pdf.

[38] W. C. Teh and K. H. Kwa, Core words and Parikh matrices, Theoret. Comput. Sci. 582 (2015), 60-69, doi:10.1016/j.tcs.2015.03.037.

[39] H. Wang, The extremal values of the Wiener index of a tree with given degree sequence, Discrete Appl. Math. 156 (2008), 2647-2654, doi:10.1016/j.dam.2007.11.005.

[40] H. Wiener, Structural determination of paraffin boiling points, J. Am. Chem. Soc. 69 (1947), 17-20, doi:10.1021/ja01193a005. 